\title{
X-ray hardness ratios for stars of different spectral types
}

\author{
E. J. A. Meurs ${ }^{1}$, P. Casey ${ }^{1}$ and L. Norci ${ }^{2}$ \\ ${ }^{1}$ Dunsink Observatory, Dublin Institute for Advanced Studies, Castleknock, Dublin 15, Ireland \\ ${ }^{2}$ School of Physics, Dublin City University, Dublin, Ireland
}

\begin{abstract}
A substantial number of stars was detected in the ROSAT All Sky Survey. Analysis of these data indicates a systematic shift in the distribution of their X-ray hardness ratios, from late type dwarfs to late type giants to early type stars, that can be attributed to systematic differences in line-of-sight absorption.
\end{abstract}

Keywords. Stars: X-rays

Extensive surveys with the Einstein and ROSAT satellites have established that X-ray emission is a common feature of normal stars. The stellar X-ray characteristics separate basically into OB stars, as a result of shocks in their strong stellar winds, and FGKM stars, as a result of coronal processes. It is uncertain whether the A stars are X-ray emitters of any importance (Haisch et al. 1992; Berghöfer et al. 1994).

The two different processes of X-ray emission in normal stars are, in principle, likely to be reflected in X-ray spectral differences. Using the ROSAT All Sky Survey (RASS) data, Motch et al. (1998) showed that normal stars of all types exhibit a broad distribution in a Hardness Ratio - Hardness Ratio diagram. However, Meurs et al. (2005) found that OB stars tend to be found at one extreme end of this overall distribution. We decided therefore to conduct a more comprehensive investigation of stellar HRs on the basis of the RASS.

The RASS produced the biggest catalogue of X-ray sources, detected at soft X-rays $(0.1-2.4 \mathrm{keV})$. We use the Bright Source Catalogue, whith count rates $>0.05 \mathrm{ct} \mathrm{s}^{-1}$. Compared to the deeper RASS Faint Source Catalogue, this choice restricts (i) contamination by other source categories and (ii) large errors on the HRs.

Lists of stars were taken from the Yale Bright Star Catalogue (Hoffleit \& Warren 1991) as available in SIMBAD. The spectral types O-M were divided in luminosity classes I, III and V. ROSAT detections were collected, based on positional coincidence within 1 arcmin; a comparison with the diagram in Motch et al. (1998) shows that conceivable misidentifications are insignificant.

The detected stars have ROSAT HRs defined as:

$$
\begin{aligned}
& \mathrm{HR} 1=((0.5-2.0)-(0.1-0.4)) /((0.5-2.0)+(0.1-0.4)) \\
& \mathrm{HR} 2=((0.9-2.0)-(0.5-0.9)) /((0.9-2.0)+(0.5-0.9))
\end{aligned}
$$

where the intervals between parentheses are in keV. If at least one of the two HRs has errors $>0.4$ the measurement is discarded, which removes effectively occasional outliers in HR1-HR2 plots.

The results of this excercise confirm a segregation for the HRs of OB and F-M stars. The OB group includes, for the B types, only B0-B2 since there is evidence that a change in behaviour occurs after B2 (Meurs et al. 1992; Berghöfer et al. 1997). 
Median Plot for Late Type (FGKM) Dwarf \& Giant stars. and $\mathrm{O}-\mathrm{B}(\mathrm{O}-2)$ stars, with error in mean

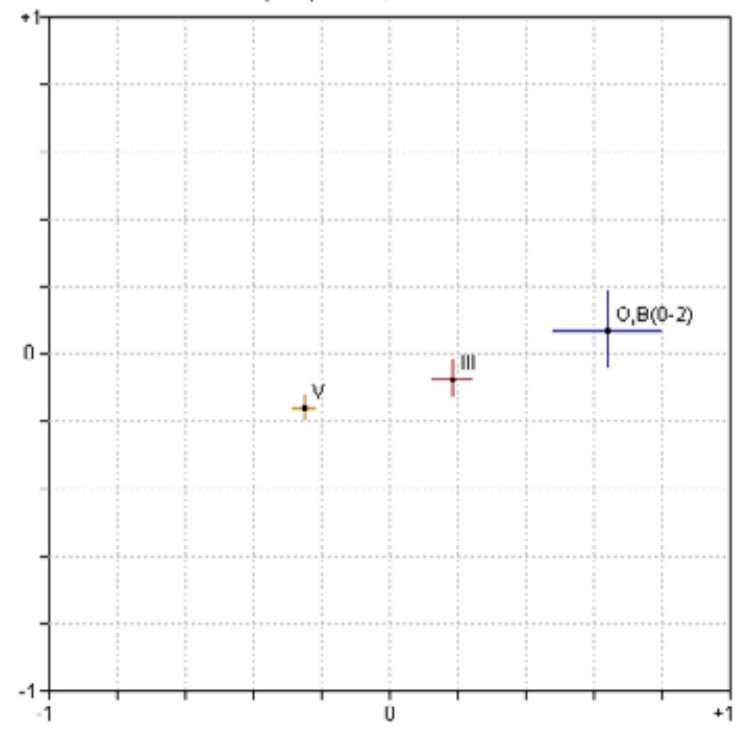

Figure 1. Median Hardness Ratios for late type dwarfs, late type giants and early type stars (from left to right).

In addition, the $\mathrm{F}-\mathrm{M}$ dwarfs and giants occupy different ranges, mostly in HR1 and to a lesser extent in HR2. The centroids for the OB stars and the late-type giants and dwarfs are plotted in Figure 1, with the standard deviations for each of these cases.

Comparing with a $\mathrm{N}_{H}$-T grid in HR1-HR2 space for thermal spectral models (Carr 2003), the shift of centroids implies a greater difference in absorbing column $\mathrm{N}_{H}$ than in temperature T. For the case of Einstein data the effect of absorption on the HR was doubted (see e.g. Vaiana 1990), but the ROSAT data are in a softer window and are more strongly sensitive to line-of-sight absorption. The progressive shift of the centroids (from late-type dwarfs via late-type giants to $\mathrm{OB}$ stars) corresponds to an increase in luminosity, thus also of increasing average distance and of absorbing column $\mathrm{N}_{H}$ (which is always most noticeable in HR1). The actual increases in $\mathrm{N}_{H}$, as deduced from the grid for thermal models, imply a typical absorption of $0.5 \mathrm{magn} / \mathrm{kpc}$, which is a realistic value. Thus, differences in $\mathrm{N}_{H}$ may be mainly responsible for the $\mathrm{OB}$ versus $\mathrm{F}-\mathrm{M}$ stars separation, rather than different X-ray emission processes as such.

\section{References}

Berghöfer, T. W., et al. 1994, A\&A 292, L5

Berghöfer, T. W., et al. 1997, A\&A 322, 167

Carr, M. 2003, PhD Thesis, Trinity College Dublin, Dublin, Ireland

Haisch, B., et al. 1992, Nature 360, 239

Hoffleit, D. \& Warren, W. H. Jr 1991, 5th "Bright Star Catalogue", Yale University Press

Meurs, E. J. A., et al. 1992, A\&A 265, L41

Meurs, E. J. A., et al. 2005, ApJ 624, 307

Motch, C., et al. 1998, AAS 132, 341

Vaiana, G. S. 1990, in "Imaging X-ray Astronomy", M. Elvis (Ed.), CUP, p.61 